DSFTH $11 / 98$

June, 1998

\title{
ON OFF-SHELL BOSONIC STRING AMPLITUDES
}

\author{
Luigi Cappiello, Raffaele Marotta \\ Roberto Pettorino and Franco Pezzella H $^{\text {t }}$ \\ Dipartimento di Scienze Fisiche, Università di Napoli \\ and I.N.F.N., sezione di Napoli \\ Mostra d'Oltremare, pad. 19, I-80125 Napoli, Italy
}

\begin{abstract}
We give a simple prescription for computing, in the framework of the bosonic string theory, off-shell one-loop amplitudes with any number of external massless particles, for both the open and the closed string. We discuss their properties and, in particular, for the two-string one-loop amplitudes we show their being transverse.
\end{abstract}

${ }^{1}$ email: Name.Surname@na.infn.it 
Perturbative string theories rely on prescriptions for computing scattering amplitudes involving on-shell physical external states.

String amplitudes are expressed as an integral over the moduli space of a punctured Riemann surface and the integrand is a correlator of vertex operators corresponding to the external states inserted at the punctures.

In on-shell string amplitudes the vertex operators are always primary fields of the underlying conformal field theory and it can be shown [1] [2] that such amplitudes are independent of the choice of a local coordinate system at the punctures. This choice is instead crucial for off-shell string amplitudes. In this case the vertex operators are nonprimary fields and their correlators turn out to be dependent on which local holomorphic coordinate system is used.

Off-shell continuations have been studied a great deal until now [3] $\div$ [] and different prescriptions have been given according to the pursued approaches.

There are important reasons for studying off-shell amplitudes, firstly the low energy limit $\left(\alpha^{\prime} \rightarrow 0\right)$ of string theories. This limit has to reproduce perturbative aspects of the ordinary gauge field theories describing the fundamental interactions, including gravity. Moreover it provides a very powerful computational tool: instead of calculating field theory amplitudes by the conventional Feynman diagrams, it is easier to calculate the corresponding string ones, that have a more compact expression and are much fewer, and then perform their low-energy limit [7] [8]. In particular closed strings can be used to shed light on perturbative quantum gravity and its divergencies [9]. In fact, perturbative computations in gravity are known to be algebraically very complex and calculations of gravity amplitudes with more than two loops [10] [11] have never been performed.

In this respect it could be very interesting to analyse the $\alpha^{\prime} \rightarrow 0$ limit of closed string amplitudes for massless states in the one-loop case. Indeed for the two-point function one should be able to make a comparison with the well-known one-loop counterterms in quantum gravity [12]. Moreover we can use the purely bosonic string since we can deal with the tachyonic divergences as in the ref. [8].

Two steps are needed: first, to give a reliable prescription for off-shell closed string 
amplitudes; second, to consider, at one loop level, the sum of the amplitude on the torus and the one on the Klein bottle, in order to reproduce results in a theory where the antisymmetric tensor is decoupled from the graviton and the dilaton.

These are our main motivations; however it is by now evident that there are also reasons of interest in off-shell string amplitudes per se as, for instance, processes involving closed string exchange among D-branes suggest [13] [14] [15].

In this letter, we limit ourselves to the first step indicated above, and, in the framework of the operatorial formalism, we give a general prescription for computing off-shell oneloop string amplitudes with any number $N$ of external massless particles. We find a simple choice of the local coordinates at the punctures on the Riemann surface, such that none of the on-shell conditions on the external states has to be kept. The string amplitude obtained in the case $N=2$ turns out to satisfy the transversality condition.

Our starting point is the $N$-string $g$-loop Vertex for the oriented bosonic closed string |11]:

$$
\begin{gathered}
V_{N ; g}=\mathcal{C}_{g} \prod_{i=1}^{N}\left[i<p_{i} ; O_{a}, O_{\bar{a}} \mid\right] \delta\left(\sum_{i=1}^{N} p_{i}\right) \int[\mathrm{d} m]_{g}^{N} \hat{V}_{N ; 0} \hat{\bar{V}}_{N ; 0} \\
\times \exp \left\{-\frac{1}{2} \sum_{i, j=1}^{N} \oint_{0} d z \oint_{0} d y \partial X^{(i)}(z) \log \frac{E\left(V_{i}(z), V_{j}(y)\right)}{V_{i}(z)-V_{j}(y)} \partial X^{(j)}(y)\right\} \\
\times \exp \left\{-\frac{1}{2} \sum_{i, j=1}^{N} \oint_{0} d \bar{z} \oint_{0} d \bar{y} \bar{\partial} \bar{X}^{(i)}(\bar{z}) \log \frac{E\left(\bar{V}_{i}(z), \bar{V}_{j}(y)\right)}{\bar{V}_{i}(z)-\bar{V}_{j}(y)} \bar{\partial} \bar{X}^{(j)}(\bar{y})\right\} \\
\times \exp \left\{i \prod_{\mu=1}^{g}\left[d^{D} k_{\mu}\right] \exp \left\{-\alpha^{\prime} \sum_{\mu, \nu=1}^{g} k_{\mu}(\pi \operatorname{I} m \tau)_{\mu \nu} k_{\nu}\right\}\right. \\
\left.\times \sqrt{\frac{\alpha^{\prime}}{2}} \sum_{i=1}^{N} \sum_{\mu=1}^{g} k_{\mu}\left[\oint_{0} d z \partial X^{(i)}(z)\left(\int_{z_{0}}^{V_{i}(z)} \omega^{\mu}\right)+\oint_{0} d \bar{z} \bar{\partial} \bar{X}^{(i)}(\bar{z})\left(\int_{\bar{z}_{0}}^{\bar{V}_{i}(z)} \bar{\omega}^{\mu}\right)\right]\right\}
\end{gathered}
$$

where $\hat{V}_{N ; 0}$ is given by

$$
\begin{aligned}
\hat{V}_{N ; 0}=\exp \{- & \left.\frac{1}{2} \sum_{\substack{i, j=1 \\
i \neq j}}^{N} \oint d z \oint d y \partial X^{(i)}(z) \log \left[V_{i}(z)-V_{j}(y)\right] \partial X^{(j)}(y)\right\} \\
& \times \exp \left\{\frac{i}{2} \oint d z \partial X^{(i)}(z) \alpha_{0}^{(i)} \log V_{i}^{\prime}(z)\right\}
\end{aligned}
$$


with an analogous definition for $\hat{\bar{V}}_{N ; 0}$. In (11) $[\mathrm{d} m]_{g}^{N}$ is the measure of integration on the moduli space for a closed Riemann surface of genus $g$. It includes the whole ghost contribution [16]. $\mathcal{C}_{g}$ is a normalization constant depending on the genus of the surface [17. The fundamental lenght $\sqrt{2 \alpha^{\prime}}$ has been extracted from the string coordinates in order to introduce the dimensionless fields:

$$
X^{\mu}(z, \bar{z})=\frac{1}{2}\left[X^{\mu}(z)+\bar{X}^{\mu}(\bar{z})\right]
$$

with

$$
\begin{gathered}
X^{\mu}(z)=q^{\mu}-i \alpha_{0}^{\mu} \log z+i \sum_{n \neq 0} \frac{\alpha_{n}^{\mu}}{n} z^{-n} \\
\bar{X}^{\mu}(\bar{z})=q^{\mu}-i \bar{\alpha}_{0}^{\mu} \log \bar{z}+i \sum_{n \neq 0} \frac{\bar{\alpha}_{n}^{\mu}}{n} \bar{z}^{-n}
\end{gathered}
$$

being $\alpha_{0}$ and $\bar{\alpha}_{0}$ defined by:

$$
\alpha_{0}^{\mu}=\bar{\alpha}_{0}^{\mu}=\sqrt{\frac{\alpha^{\prime}}{2}} p^{\mu}
$$

In the operator formalism $V_{N ; g}$, as defined in Eq. (1), provides an efficient way of computing scattering amplitudes among arbitrary states at all orders in perturbation theory. In fact, by saturating the operator $V_{N ; g}$ with $N$ external states $\left|\alpha_{1}>\cdots\right| \alpha_{N}>$, the corresponding amplitude is obtained:

$$
A^{(g)}\left(\alpha_{1}, \ldots \alpha_{N}\right)=V_{N ; g}\left|\alpha_{1}>\cdots\right| \alpha_{N}>
$$

The Vertex $V_{N ; g}$ depends on the $N$ complex Koba-Nielsen variables $z_{i}$ 's corresponding to the punctures of the external states, through $N$ conformal transformations $V_{i}(z)$ 's , which define a local coordinate system vanishing around each $z_{i}$, i.e.:

$$
V_{i}(0)=z_{i}
$$

When $V_{N ; g}$ is saturated with $N$ physical string states satisfying the mass-shell condition, the corresponding amplitude does not depend on the $V_{i}$ 's. If this condition is 
relaxed, the dependence of $V_{N ; g}$ on them is transferred to the off-shell amplitude. This is analogous to what happens in gauge theories, where on-shell amplitudes are gauge invariant, while their off-shell counterparts are not.

Let us concentrate on the case in which all the $N$ external states belong to the massless level of the bosonic closed string; let $\mid \epsilon ; p>$ be such a state with polarization $\epsilon$ and momentum $p$ :

$$
\left|\epsilon ; p>=\mathcal{N}_{0} \epsilon_{\mu \nu} \alpha_{-1}^{\mu} \bar{\alpha}_{-1}^{\nu}\right| p>
$$

Depending on the symmetry properties of the polarization $\epsilon_{\mu \nu}$, this state defines the antisymmetric tensor or a combination of graviton and dilaton states. In our notations the normalization factor is $\mathcal{N}_{0}=\kappa / \pi$, where $\kappa$ is the gravitational constant in $d$ dimensions.

An on-shell massless state satisfies the conditions:

$$
p^{2}=0 \quad \epsilon \cdot p=0
$$

and it is defined through its corresponding vertex operator, which is a primary field of the underlying conformal field theory:

$$
\left|\epsilon ; p>=\lim _{z, \bar{z} \rightarrow 0} V(z, \bar{z})\right| 0>
$$

with

$$
V(z, \bar{z})=i \mathcal{N}_{0} \epsilon_{\mu \nu}: \partial_{z} X^{\mu}(z) \partial_{\bar{z}} \bar{X}^{\nu}(\bar{z}) e^{i \sqrt{2 \alpha^{\prime}} p \cdot X(z, \bar{z})}:
$$

Since we are interested in off-shell amplitudes, we will release the conditions (9) and we will write down the expression of $V_{N ; g}$ ready to be saturated with such states; we denote it as $\mathcal{V}_{N ; g}$ and it is given by:

$$
\begin{aligned}
\mathcal{V}_{N ; g}=\mathcal{C}_{g}<\Omega \mid \int[\mathrm{d} m]_{N}^{g} & \exp \left\{\left.\frac{1}{2} \sum_{i=1}^{N} \sqrt{\frac{\alpha^{\prime}}{2}} p^{(i)} \cdot\left[\sqrt{\frac{\alpha^{\prime}}{2}} p^{(i)}+\alpha_{1}^{(i)} \partial_{z}+\bar{\alpha}_{1}^{(i)} \partial_{\bar{z}}\right] \log \left|V_{i}^{\prime}(z)\right|^{2}\right|_{z=0}\right\} \\
& \times \exp \left\{\sum_{\substack{i, j=1 \\
i \neq j}}^{N}\left[\sqrt{\frac{\alpha^{\prime}}{2}} p^{(i)}+\alpha_{1}^{(i)} V_{i}^{\prime}(0) \partial_{z_{i}}+\bar{\alpha}_{1}^{(i)} \bar{V}_{i}^{\prime}(0) \partial_{\bar{z}_{i}}\right]\right.
\end{aligned}
$$




$$
\begin{aligned}
& \left.\cdot\left[\sqrt{\frac{\alpha^{\prime}}{2}} p^{(j)}+\alpha_{1}^{(j)} V_{j}^{\prime}(0) \partial_{z_{j}}+\bar{\alpha}_{1}^{(j)} \bar{V}_{j}^{\prime}(0) \partial_{\bar{z}_{j}}\right] \mathcal{G}\left(z_{i}, z_{j}\right)\right\} \\
& \times \exp \left\{-\left.2 \sum_{i=1}^{N} \alpha_{1}^{(i)} \cdot \bar{\alpha}_{1}^{(i)}\left|V_{i}^{\prime}(0)\right|^{2} \partial_{z} \partial_{\bar{z}} \mathcal{G}\left(z, z_{i}\right)\right|_{z=z_{i}}\right\}
\end{aligned}
$$

where

$$
\mathcal{G}\left(z_{i}, z_{j}\right) \equiv \frac{1}{2} \log \left|E\left(z_{i}, z_{j}\right)\right|^{2}-\frac{1}{2}\left(\operatorname{Re} \int_{z_{i}}^{z_{j}} \omega^{\mu}\right)(2 \pi \operatorname{I} m \tau)_{\mu \nu}^{-1}\left(\operatorname{Re} \int_{z_{i}}^{z_{j}} \omega^{\nu}\right)
$$

is the $g$-loop world-sheet bosonic Green function [18].

The expression of $\mathcal{V}_{N ; g}$ in (12) has been obtained by performing the gaussian integration over the internal momenta $k_{\mu}$ 's. It has generated the term, written in the last line, in which right and left movers are mixed; in other words, the vertex is not holomorphically factorized.

We can exploit the freedom of choosing the conformal local maps $V_{i}(z)$ 's in order to write $\mathcal{V}_{N ; g}$ in a more geometrical form depending only on the Green function. First we rescale this latter as follows:

$$
\begin{aligned}
G\left(z_{i}, z_{j}\right) & =\mathcal{G}\left(z_{i}, z_{j}\right)-\frac{1}{4} \log \left|V_{i}^{\prime}(0) V_{j}^{\prime}(0)\right|^{2} \\
& =\frac{1}{2} \log \frac{\left|E\left(z_{i}, z_{j}\right)\right|^{2}}{\left|V_{i}^{\prime}(0) V_{j}^{\prime}(0)\right|}-\frac{1}{2}\left(\operatorname{Re} \int_{z_{i}}^{z_{j}} \omega^{\mu}\right)(2 \pi \operatorname{I} m \tau)_{\mu \nu}^{-1}\left(\operatorname{Re} \int_{z_{i}}^{z_{j}} \omega^{\nu}\right)
\end{aligned}
$$

This expression coincides with the one given in literature [20] [21], where the conformal maps $V_{i}(z)$ 's may depend on all the moduli of the world sheet and on the positions of the punctures.

In the following we restrict ourselves to the $g=1$ loop case and, at this order, a choice corresponding to

$$
V_{i}^{\prime}(0)=z_{i}
$$

reproduces the translational invariant Green function on the torus [18. However a careful inspection of the expression (12) shows that the condition (15) is not sufficient to write the vertex $\mathcal{V}_{N ; 1}$ only in terms of the Green function; we are then led to impose the following further condition:

$$
V_{i}^{\prime \prime}(0)=z_{i}
$$


The constraints (77), (15) and (16) may be satisfied, choosing, for instance, the following holomorphic local coordinate map at the puncture $z_{i}$ :

$$
V_{i}(z)=z_{i} e^{z}
$$

This choice allows us to rewrite $\mathcal{V}_{N ; 1}$ as:

$$
\begin{aligned}
\mathcal{V}_{N ; 1}=\mathcal{C}_{1} & <\Omega \mid \int[\mathrm{d} m]_{N}^{1} \exp \left\{\sum_{\substack{i, j=1 \\
i \neq j}}^{N}\left[\sqrt{\frac{\alpha^{\prime}}{2}} p^{(i)}+\alpha_{1}^{(i)} z_{i} \partial_{z_{i}}+\bar{\alpha}_{1}^{(i)} \bar{z}_{i} \partial_{\bar{z}_{i}}\right]\right. \\
& \left.\cdot\left[\sqrt{\frac{\alpha^{\prime}}{2}} p^{(j)}+\alpha_{1}^{(j)} z_{j} \partial_{z_{j}}+\bar{\alpha}_{1}^{(j)} \bar{z}_{j} \partial_{\bar{z}_{j}}\right] G\left(z_{i}, z_{j}\right)\right\} \\
& \times \exp \left\{-\left.2 \sum_{i=1}^{N} \alpha_{1}^{(i)} \cdot \bar{\alpha}_{1}^{(i)}\left|z_{i}\right|^{2} \partial_{z} \partial_{\bar{z}} G\left(z, z_{i}\right)\right|_{z=z_{i}}\right\}
\end{aligned}
$$

and it reproduces, for small values of $z$, the gauge

$$
V_{i}(z)=z_{i} z+z_{i}
$$

that has been proposed for the open string [8]. We point out, however, that in this case, the map (19) has to be considered together with the condition $\epsilon \cdot p=0$ for external photons even if off mass-shell. Differently, our choice (17) does not need to be coupled to any other condition: our proposal for the local maps $V_{i}(z)$ 's includes all the off-shell conditions. In the following we will check for the two-gluon amplitude that this prescription also works in the open string case.

The vertex $\mathcal{V}_{N ; 1}(18)$ is a very closed expression that gives the possibility of easily computing off-shell one-loop amplitudes for an arbitrary number of external massless states. Due to the structure of the closed string states (8), one can think at the polarization tensor as:

$$
\epsilon_{\mu \nu}=\xi_{\mu} \otimes \bar{\xi}_{\nu}
$$

Therefore it is straightforward writing the $N$-string one-loop amplitude:

$$
A_{N ; 1}=\mathcal{C}_{1} \int[\mathrm{d} m]_{N}^{1} \exp \left\{\sum_{\substack{i, j=1 \\ i \neq j}}^{N}\left[\sqrt{\frac{\alpha^{\prime}}{2}} p^{(i)}+\xi^{(i)} z_{i} \partial_{z_{i}}+\bar{\xi}^{(i)} \bar{z}_{i} \partial_{\bar{z}_{i}}\right]\right.
$$




$$
\begin{aligned}
& \left.\cdot\left[\sqrt{\frac{\alpha^{\prime}}{2}} p^{(j)}+\xi^{(j)} z_{j} \partial_{z_{j}}+\bar{\xi}^{(j)} \bar{z}_{j} \partial_{\bar{z}_{j}}\right] G\left(z_{i}, z_{j}\right)\right\} \\
& \times \exp \left\{-\left.2 \sum_{i=1}^{N} \xi^{(i)} \cdot \bar{\xi}^{(i)}\left|z_{i}\right|^{2} \partial_{z} \partial_{\bar{z}} G\left(z, z_{i}\right)\right|_{z=z_{i}}\right\} .
\end{aligned}
$$

This expression has to be understood as an expansion in $\xi^{(i)}$ and $\bar{\xi}^{(i)}$ restricted only to the linear terms.

In order to check the properties of the off-shell amplitudes obtained through the vertex (18), we consider the case $N=2$.

The normalization factor is given by $\mathcal{C}_{1}=\left(2 \pi \alpha^{\prime}\right)^{-d / 2}$ [17]. The one-loop measure is

$$
[\mathrm{d} m]_{2}^{1}=\frac{d^{2} z_{1} d^{2} z_{2}}{\left|z_{1}\right|^{2}\left|z_{2}\right|^{2}} \frac{d^{2} k}{|k|^{4}}[-\ln |k|]^{-d / 2} \prod_{n=1}^{+\infty}\left(\left|1-k^{n}\right|^{2}\right)^{2-d} .
$$

and the explicit expression of the Green function (13) is [18]:

$$
\mathcal{G}\left(z_{i}, z_{j}\right)=\frac{1}{2} \log \left|\left(z_{i}-z_{j}\right) \prod_{n=1}^{+\infty} \frac{\left(z_{i}-k^{n} z_{j}\right)\left(z_{j}-k^{n} z_{i}\right)}{z_{i} z_{j}\left(1-k^{n}\right)^{2}}\right|^{2}+\frac{1}{2} \frac{\log ^{2}\left|z_{1} / z_{2}\right|}{\log |k|} .
$$

One-loop two-string amplitudes can be obtained by expanding $A_{2 ; 1}$ up to terms linear in $\xi^{(1) \mu} \bar{\xi}^{(1) \nu} \xi^{(2) \rho} \bar{\xi}^{(2) \sigma}$. After some algebra we get:

$$
A_{2 ; 1}=\mathcal{N}_{0}^{2} \mathcal{C}_{1} \epsilon^{\mu \nu(1)} \epsilon^{\rho \sigma(2)} T_{\mu \nu \rho \sigma}
$$

with

$$
\begin{aligned}
T_{\mu \nu \rho \sigma}= & 4\left[\eta_{\mu \nu} \eta_{\rho \sigma} a_{1}+\eta_{\mu \sigma} \eta_{\nu \rho} a_{2}+\eta_{\mu \rho} \eta_{\nu \sigma} a_{3}\right. \\
& +\alpha^{\prime}\left(\eta_{\mu \rho} p_{\sigma}^{(1)} p_{\nu}^{(2)} a_{4}-\eta_{\mu \nu} p_{\rho}^{(1)} p_{\sigma}^{(1)} a_{5}+\eta_{\mu \sigma} p_{\rho}^{(1)} p_{\nu}^{(2)} a_{6}\right. \\
& \left.+\eta_{\nu \rho} p_{\sigma}^{(1)} p_{\mu}^{(2)} a_{7}-\eta_{\rho \sigma} p_{\mu}^{(2)} p_{\nu}^{(2)} a_{8}+\eta_{\nu \sigma} p_{\rho}^{(1)} p_{\mu}^{(2)} a_{9}\right) \\
& \left.+\left(\alpha^{\prime}\right)^{2} p_{\rho}^{(1)} p_{\nu}^{(1)} p_{\mu}^{(2)} p_{\sigma}^{(2)} a_{10}\right]
\end{aligned}
$$

where the coefficients $a_{i}{ }^{\prime}$ s, $i=1, \ldots, 10$, are given by: 


$$
\begin{aligned}
& a_{1}=\int[d m]_{2}^{1} e^{\alpha^{\prime} p^{(1)} \cdot p^{(2)} G\left(z_{1}, z_{2}\right)}\left|z_{1}\right|^{2}\left|z_{2}\right|^{2} \partial_{z_{1}} \partial_{\bar{z}_{1}} G\left(z_{1}, z_{2}\right) \partial_{z_{2}} \partial_{\bar{z}_{2}} G\left(z_{1}, z_{2}\right) \\
& a_{2}=\int[d m]_{2}^{1} e^{\alpha^{\prime} p^{(1)} \cdot p^{(2)} G\left(z_{1}, z_{2}\right)}\left|z_{1}\right|^{2}\left|z_{2}\right|^{2} \partial_{z_{1}} \partial_{\bar{z}_{2}} G\left(z_{1}, z_{2}\right) \partial_{\bar{z}_{1}} \partial_{z_{2}} G\left(z_{1}, z_{2}\right) \\
& a_{3}=\int[d m]_{2}^{1} e^{\alpha^{\prime} p^{(1)} \cdot p^{(2)} G\left(z_{1}, z_{2}\right)}\left|z_{1}\right|^{2}\left|z_{2}\right|^{2} \partial_{z_{1}} \partial_{z_{2}} G\left(z_{1}, z_{2}\right) \partial_{\bar{z}_{1}} \partial_{\bar{z}_{2}} G\left(z_{1}, z_{2}\right) \\
& a_{4}=\int[d m]_{2}^{1} e^{\alpha^{\prime} p^{(1)} \cdot p^{(2)} G\left(z_{1}, z_{2}\right)}\left|z_{1}\right|^{2}\left|z_{2}\right|^{2} \partial_{z_{1}} \partial_{z_{2}} G\left(z_{1}, z_{2}\right) \partial_{\bar{z}_{1}} G\left(z_{1}, z_{2}\right) \partial_{\bar{z}_{2}} G\left(z_{1}, z_{2}\right) \\
& a_{5}=\int[d m]_{2}^{1} e^{\alpha^{\prime} p^{(1)} \cdot p^{(2)} G\left(z_{1}, z_{2}\right)}\left|z_{1}\right|^{2}\left|z_{2}\right|^{2} \partial_{z_{1}} \partial_{\bar{z}_{1}} G\left(z_{1}, z_{2}\right) \partial_{z_{2}} G\left(z_{1}, z_{2}\right) \partial_{\bar{z}_{2}} G\left(z_{1}, z_{2}\right) \\
& a_{6}=\int[d m]_{2}^{1} e^{\alpha^{\prime} p^{(1)} \cdot p^{(2)} G\left(z_{1}, z_{2}\right)}\left|z_{1}\right|^{2}\left|z_{2}\right|^{2} \partial_{z_{1}} \partial_{\bar{z}_{2}} G\left(z_{1}, z_{2}\right) \partial_{\bar{z}_{1}} G\left(z_{1}, z_{2}\right) \partial_{z_{2}} G\left(z_{1}, z_{2}\right) \\
& a_{7}=\int[d m]_{2}^{1} e^{\alpha^{\prime} p^{(1)} \cdot p^{(2)} G\left(z_{1}, z_{2}\right)}\left|z_{1}\right|^{2}\left|z_{2}\right|^{2} \partial_{\bar{z}_{1}} \partial_{z_{2}} G\left(z_{1}, z_{2}\right) \partial_{z_{1}} G\left(z_{1}, z_{2}\right) \partial_{\bar{z}_{2}} G\left(z_{1}, z_{2}\right) \\
& a_{8}=\int[d m]_{2}^{1} e^{\alpha^{\prime} p^{(1)} \cdot p^{(2)} G\left(z_{1}, z_{2}\right)}\left|z_{1}\right|^{2}\left|z_{2}\right|^{2} \partial_{z_{2}} \partial_{\bar{z}_{2}} G\left(z_{1}, z_{2}\right) \partial_{z_{1}} G\left(z_{1}, z_{2}\right) \partial_{\bar{z}_{1}} G\left(z_{1}, z_{2}\right) \\
& a_{9}=\int[d m]_{2}^{1} e^{\alpha^{\prime} p^{(1)} \cdot p^{(2)} G\left(z_{1}, z_{2}\right)}\left|z_{1}\right|^{2}\left|z_{2}\right|^{2} \partial_{\bar{z}_{1}} \partial_{\bar{z}_{2}} G\left(z_{1}, z_{2}\right) \partial_{z_{1}} G\left(z_{1}, z_{2}\right) \partial_{z_{2}} G\left(z_{1}, z_{2}\right) \\
& a_{10}=\int[d m]_{2}^{1} e^{\alpha^{\prime} p^{(1)} \cdot p^{(2)} G\left(z_{1}, z_{2}\right)}\left|z_{1}\right|^{2}\left|z_{2}\right|^{2} \partial_{z_{1}} G\left(z_{1}, z_{2}\right) \partial_{\bar{z}_{1}} G\left(z_{1}, z_{2}\right) \partial_{z_{2}} G\left(z_{1}, z_{2}\right) \partial_{\bar{z}_{2}} G\left(z_{1}, z_{2}\right)
\end{aligned}
$$

While these coefficients can be computed in the limit $\alpha^{\prime} \rightarrow 0$ (and we will give the results in a forthcoming paper), some of their properties can be proved on general grounds. By integrating by parts all the terms containing double derivatives of the Green function, it can be shown that only three $a_{i}$ 's are independent. We would like here to stress that at the one-loop order it is possible, due to the properties of the Green function on the torus, to disregard surface terms coming from the integration by parts. Hence this case results to be even easier than the tree level.

Indeed, at one-loop order the expression for $T_{\mu \nu \rho \sigma}$ drastically simplifies reducing to:

$$
\begin{aligned}
T_{\mu \nu \rho \sigma}= & \left\{-\frac{2}{p^{2}}\left(a_{3}+a_{2}\right)\left[\eta_{\mu \rho} p_{\nu} p_{\sigma}+\eta_{\nu \rho} p_{\mu} p_{\sigma}+\eta_{\mu \sigma} p_{\rho} p_{\nu}+\eta_{\nu \sigma} p_{\rho} p_{\mu}\right]\right. \\
& -\frac{4}{p^{2}} a_{1}\left[\eta_{\mu \nu} p_{\rho} p_{\sigma}+\eta_{\rho \sigma} p_{\mu} p_{\nu}\right]+\frac{4}{p^{4}}\left(a_{1}+a_{2}+a_{3}\right) p_{\mu} p_{\nu} p_{\rho} p_{\sigma} \\
& \left.+2\left(a_{3}+a_{2}\right)\left[\eta_{\mu \rho} \eta_{\nu \sigma}+\eta_{\nu \rho} \eta_{\mu \sigma}\right]+4 a_{1} \eta_{\mu \nu} \eta_{\rho \sigma}\right\} \\
& +\left\{-\frac{2}{p^{2}}\left(a_{3}-a_{2}\right)\left[\eta_{\mu \rho} p_{\nu} p_{\sigma}-\eta_{\nu \rho} p_{\mu} p_{\sigma}+\eta_{\nu \sigma} p_{\mu} p_{\rho}-\eta_{\mu \sigma} p_{\rho} p_{\nu}\right]\right. \\
& \left.+2\left(a_{3}-a_{2}\right)\left[\eta_{\mu \rho} \eta_{\nu \sigma}-\eta_{\mu \sigma} \eta_{\nu \rho}\right]\right\} \equiv S_{\mu \nu \rho \sigma}+A_{\mu \nu \rho \sigma}
\end{aligned}
$$

where we used the momentum conservation for setting $p^{(1)}=-p^{(2)} \equiv p$ and made explicit the symmetry properties on the indices $(\mu \nu)$ which refer to the polarization tensor of the 
particle $(1)$ and $(\rho \sigma)$ which refer to the one of the particle (2). Of course the amplitude is symmetric under the exchange of the states (1) and (2).

We notice that the symmetric part $S_{\mu \nu \rho \sigma}$ depends only on the two quantities $a_{1}$ and $a_{2}+a_{3}$, while the antisymmetric one $A_{\mu \nu \rho \sigma}$ has an overall factor $a_{3}-a_{2}$.

The one loop amplitude, as an off-shell string amplitude, has the very remarkable property of being transverse:

$$
p^{\mu} T_{\mu \nu \rho \sigma}=0
$$

In this respect the relations among the quantities $a_{i}$ 's play the role of Ward identities for the two-point function, which in field theory follow from the gauge invariance (i.e. invariance under general coordinates transformations). However in quantum gravity transversality does not always hold; it does, for instance, when background-field techniques are used [19.

In the final part of this work we will check that our prescription (17) also works in the open string case [8].

The starting point is given by the $N$-string $g$-loop Vertex for the open string [1]:

$$
\begin{gathered}
V_{N ; g}=\mathcal{C}_{g}^{o p e n} \prod_{i=1}^{N}\left[{ }_{i}<p_{i} ; O_{a} \mid\right] \delta\left(\sum_{i=1}^{N} p_{i}\right) \int[\mathrm{d} m]_{g}^{N} \hat{V}_{N ; 0} \\
\times \exp \left\{-\frac{1}{2} \sum_{i, j=1}^{N} \oint_{0} d z \oint_{0} d y \partial X^{(i)}(z) \log \frac{E\left(V_{i}(z), V_{j}(y)\right)}{V_{i}(z)-V_{j}(y)} \partial X^{(j)}(y)\right\} \\
\times \int \prod_{\mu=1}^{g}\left[d^{D} k_{\mu}\right] \exp \left\{-\alpha^{\prime} \sum_{\mu, \nu=1}^{g} k_{\mu}(2 \pi \mathrm{I} m \tau)_{\mu \nu} k_{\nu}+i \sqrt{2 \alpha^{\prime}} \sum_{i=1}^{N} \sum_{\mu=1}^{g} k_{\mu}\left[\oint_{0} d z \partial X^{(i)}(z)\left(\int_{z_{0}}^{V_{i}(z)} \omega^{\mu}\right)\right]\right\}
\end{gathered}
$$

with $\alpha_{0}^{\mu}=\sqrt{2 \alpha^{\prime}} p^{\mu}$ and $\hat{V}_{N ; 0}$ defined in (2). From (22) one can have the corresponding expression for $N$ external "photons". It is given by:

$$
\begin{aligned}
& \mathcal{V}_{N ; g}=\mathcal{C}_{g}^{o p e n}<\Omega \mid \int[\mathrm{d} m]_{N}^{g} \exp \left\{\left.\frac{1}{2} \sum_{i=1}^{N} \sqrt{2 \alpha^{\prime}} p^{(i)} \cdot\left[\sqrt{2 \alpha^{\prime}} p^{(i)}+\alpha_{1}^{(i)} \partial_{z}\right] \log V_{i}^{\prime}(z)\right|_{z=0}\right\} \\
& \quad \times \exp \frac{1}{2}\left\{\sum_{\substack{i, j=1 \\
i \neq j}}^{N}\left[\sqrt{2 \alpha^{\prime}} p^{(i)}+\alpha_{1}^{(i)} V_{i}^{\prime}(0) \partial_{z_{i}}\right] \cdot\left[\sqrt{2 \alpha^{\prime}} p^{(j)}+\alpha_{1}^{(j)} V_{j}^{\prime}(0) \partial_{z_{j}}\right] \mathcal{G}\left(z_{i}, z_{j}\right)\right\}
\end{aligned}
$$


where

$$
\mathcal{G}\left(z_{i}, z_{j}\right) \equiv \log E\left(z_{i}, z_{j}\right)-\frac{1}{2}\left(\int_{z_{i}}^{z_{j}} \omega^{\mu}\right)(2 \pi \operatorname{Im} \tau)_{\mu \nu}^{-1}\left(\int_{z_{i}}^{z_{j}} \omega^{\nu}\right)
$$

is the $g$-loop Green function that, also in this case, can be rescaled as:

$$
G\left(z_{i}, z_{j}\right) \equiv \log \frac{E\left(z_{i}, z_{j}\right)}{\sqrt{V_{i}^{\prime}(0) V_{j}^{\prime}(0)}}-\frac{1}{2}\left(\int_{z_{i}}^{z_{j}} \omega^{\mu}\right)(2 \pi \operatorname{I} m \tau)_{\mu \nu}^{-1}\left(\int_{z_{i}}^{z_{j}} \omega^{\nu}\right) .
$$

Once again we restrict ourself to the $g=1$ loop case. The choice (17) in (25) reproduces the right translational invariant Green function and allows us to rewrite the Vertex $\mathcal{V}_{N ; 1}$ only in terms of it:

$$
\begin{gathered}
\mathcal{V}_{N ; 1}=\mathcal{C}_{1}^{\text {open }}<\Omega \mid \int[\mathrm{d} m]_{N}^{1} \\
\times \exp \left\{\sum_{\substack{i, j=1 \\
i \neq j}}^{N}\left[\sqrt{2 \alpha^{\prime}} p^{(i)}+\alpha_{1}^{(i)} z_{i} \partial_{z_{i}}\right]\left[\sqrt{2 \alpha^{\prime}} p^{(j)}+\alpha_{1}^{(j)} z_{j} \partial_{z_{j}}\right] G\left(z_{i}, z_{j}\right)\right\} .
\end{gathered}
$$

By saturating $\mathcal{V}_{N ; 1}$ on two photon states, defined by:

$$
\left|\epsilon ; p>=\mathcal{N}_{0}^{p h .} \epsilon_{\mu} \alpha_{-1}^{\mu}\right| p>
$$

we get the corresponding amplitude:

$$
A_{2 ; 1}=\left(\mathcal{N}_{0}^{p h .}\right)^{2} \mathcal{C}_{1}^{\text {open }} \epsilon^{(1) \mu} \epsilon^{(2) \nu} T_{\mu \nu}
$$

with

$$
T_{\mu \nu}=2\left(a_{1} \eta_{\mu \nu}+4 \alpha^{\prime} a_{2} p_{\nu}^{(1)} p_{\mu}^{(2)}\right)
$$

where the coefficients $a_{i}$ 's are given by:

$$
\begin{aligned}
& a_{1}=\int[d m]_{2}^{1} e^{4 \alpha^{\prime} p^{(1)} \cdot p^{(2)} G\left(z_{1}, z_{2}\right)} z_{1} z_{2} \partial_{z_{1}} \partial_{z_{2}} G\left(z_{1}, z_{2}\right) \\
& a_{2}=\int[d m]_{2}^{1} e^{4 \alpha^{\prime} p^{(1)} \cdot p^{(2)} G\left(z_{1}, z_{2}\right)} z_{1} z_{2} \partial_{z_{1}} G\left(z_{1}, z_{2}\right) \partial_{z_{2}} G\left(z_{1}, z_{2}\right) .
\end{aligned}
$$

By integrating by parts the term containing the double derivative of $G\left(z_{i}, z_{j}\right)$, we get a relation between $a_{1}$ and $a_{2}$ :

$$
a_{1}=-4 \alpha^{\prime} p^{(1)} \cdot p^{(2)} a_{2}
$$


so that we derive for $T_{\mu \nu}$ the following expression:

$$
T_{\mu \nu}=4 \alpha^{\prime} a_{2}\left[p^{2} \eta_{\mu \nu}-p_{\nu} p_{\mu}\right]
$$

Also this amplitude, even being an off-shell string amplitude, is transverse. Once $a_{2}$ is evaluated in the $\alpha^{\prime} \rightarrow 0$ limit, the results of gauge field theory are properly reproduced [8.

In conclusion, we have given here a systematic way of obtaining off-shell one-loop amplitudes with $N$ external massless states; the same procedure can be now applied to the Klein bottle [22].

We defer to a forthcoming work a full analysis of the low energy limit of off-shell both of open and closed string amplitudes.

ACKNOWLEDGMENTS. We thank P. Di Vecchia for many helpful discussions and for useful comments on a preliminary version of the paper.

\section{References}

[1] P. Di Vecchia, F. Pezzella, M. Frau, A. Lerda, K. Hornfeck and S. Sciuto, Nucl. Phys. B322 (1989) 317.

[2] A. Neveu and P. West, Nucl. Phys. B311 (1989) 79.

[3] A. Cohen, G. Moore, P. Nelson and J. Polchinski, Nucl. Phys. B267 (1986) 143; Nucl. Phys. B281 (1987) 127.

[4] Z. Bern, D. A. Kosower and K. Roland, Nucl. Phys. B344 (1990) 309.

[5] R. C. Myers and V. Periwal, Talk given at the International Workshop on String Theory, Quantum Gravity and the Unification of Fundamental Interactions, Rome, 1992, hep-th/9211078.

[6] A. Belopolsky and B. Zwiebach, Nucl. Phys. B442 (1995) 494, hep-th/9409015. 
[7] Z. Bern and D. A. Kosower, Phys. Rev. D38 (1988) 1888; Nucl. Phys. B321 (1989) 605; Nucl. Phys. B379 (1992) 451.

[8] P. Di Vecchia, R. Marotta, A. Lerda, R. Russo and L. Magnea, Nucl. Phys. B469 (1996) 235, hep-th/9601143.

[9] Z. Bern, D. C. Dunbar and T. Shimada, Phys. Lett. B312 (1993) 277, hepth/9307001.

[10] M. H. Goroff and A. Sagnotti, Phys. Lett. B160 (1985) 81.

[11] A. E. M. van de Ven, Nucl. Phys. B378 (1992) 309.

[12] G. 't Hooft and M. Veltman, Ann. Inst. Henri Poincarè, vol. XX, n. 1, 1974, 69-94.

[13] J. Polchinski, Phys. Rev. Lett. 75 (1995) 4727, hep-th/9510017.

[14] S. Ramgoolam and L. Thorlacius, Nucl. Phys. B483 (1997) 248, hep-th/9607113.

[15] M. Billó, P. Di Vecchia, M. Frau, A. Lerda, I. Pesando, R. Russo and S. Sciuto, Microscopic string analysis of the D0-D8 brane system and dual $R$ - $R$ states, hepth/9802088.

[16] P. Di Vecchia, M. Frau, A. Lerda and S. Sciuto, Phys. Lett. B199 (1987) 49.

[17] G. Cristofano, R. Marotta and K. Roland, Nucl. Phys. B392 (1993) 345.

[18] M. Green, J. Schwarz and E. Witten, Superstring Theory, Cambridge University Press (1987).

[19] M. J. Duff, Covariant Quantization in Quantum Gravity, edited by C. J. Isham, R. Penrose and D. W. Sciama, Clarendon Press Oxford (1975).

[20] E. Martinec, Nucl. Phys. B281 (1987) 157.

[21] K. Roland and H.-T. Sato, Nucl. Phys. B480 (1996) 99, hep-th/9604152. 
[22] L. Cappiello, R. Marotta, R. Pettorino and F. Pezzella, Off-shell amplitudes for nonoriented closed strings, to appear. 